\section{Hanseníase no Estado do Espírito Santo, Brasil: uma endemia em ascensão?}

\author{
Leprosy in Espírito Santo State, Brazil: a growing \\ endemic?
}

\author{
1 Secretaria de Estado da \\ Saúde do Espírito Santo, \\ Vitória, Brasil. \\ 2 Faculdade de Saúde \\ Pública, Universidade de São \\ Paulo, São Paulo, Brasil. \\ Correspondência \\ C. L. Martins \\ Faculdade de Saúde Pública \\ Universidade de São Paulo. \\ Av. Dr. Arnaldo 715, \\ São Paulo, SP \\ 01246-904, Brasil. \\ cleide@usp.br
}

\begin{abstract}
This study provides a trend analysis of leprosy among patients in the State of Espirito Santo, Brazil, from 1980 to 2003. Using temporal series statistical models, an upward trend was identified throughout the period in the overall detection rate $(p<0.05)$, with an apparent stabilization at the end of the period. We also observed an upward trend for the following periods: (i) 1980-1987 in the $<15$ and $\geq 50$-year age groups and for paucibacillary forms; (ii) 1988-1995 for the 15-19, 20-29, and $\geq 50$-year groups and for multibacillary forms; and (iii) 1996-2003 in the 20-29-year group and paucibacillary forms. The indicators for evaluation of the endemic indicate: stable levels in grade 2 disability (mean of 6\%); a proportion of less than 10\% of cases in individuals $<15$ years of age; and a treatment dropout rate of approximately $6 \%$. Prevalence showed a sharp decline. The upward trend can be explained partially by greater surveillance sensitivity, but the high proportion of individuals $<15$ highlights the need for studies aimed at better knowledge of residual sources of infection, especially in the household.
\end{abstract}

Leprosy; Endemic Diseases; Time Series Studies
Marilda Vieira Moreira ${ }^{1}$

Eliseu Alves Waldman 2

Cleide Lavieri Martins 2

\section{Introdução}

A hanseníase ainda constitui grave problema de saúde pública, nas regiões em desenvolvimento 1 . Estudos recentes indicam que cerca de $90 \%$ dos casos vivem em seis países: Índia, Brasil, Nepal, Myanmar, Moçambique e Madagascar 2,3,4 .

Dados de 2003 mostram que a prevalência de hanseníase no Brasil situava-se em 4,5 casos/10 mil habitantes 5 , colocando nosso país e a Índia como os mais atingidos por esta endemia, em todo mundo ${ }^{3}$. No âmbito das Américas, é o único país a não atingir a meta de prevalência inferior a 1 caso/10 mil habitantes, proposta pela Organização Mundial da Saúde (OMS) 6 .

Sua distribuição no Brasil é heterogênea $4,7,8,9,10$, variando de prevalências inferiores à meta proposta pela OMS, como no Estado do Rio Grande do Sul 5, a regiões hiperendêmicas, particularmente nas regiões Norte e Nordeste 11,12,13,14. O Estado do Espírito Santo foi o único da Região Sudeste que apresentou elevados níveis de endemicidade em 2003 5, fato que o coloca entre os prioritários para o programa de controle dessa endemia, no país.

Esse estado apresentou nos últimos anos alguns resultados favoráveis acompanhando a tendência do Brasil e mundial 3,6,15,16. No entanto, à semelhança de outras regiões do Brasil 17, o Estado do Espírito Santo tem apresentado um aumento da incidência não perfeitamente explicado, fato que justifica um estudo mais detalha- 
do da trajetória da endemia nos últimos anos. O objetivo deste estudo é descrever a situação atual da hanseníase no Estado do Espírito Santo e analisar a tendência da sua incidência, durante o período de 1980 a 2003.

\section{Metodologia}

\section{Delineamento}

Estudo descritivo com análise de tendência.

\section{Área e população de estudo}

A área de estudo é o Estado do Espírito Santo, que já concluiu o processo de descentralização dos serviços de saúde iniciado em 1995, tendo o programa de controle da hanseníase acompanhado este processo. A população de estudo abrange o universo dos casos de hanseníase, notificados entre 1980 e 2003, residentes no estado.

\section{Definição de caso e conceitos}

É considerado caso de hanseníase qualquer indivíduo, que independentemente de sexo e idade, apresente uma ou mais de uma das seguintes características: presença de lesão(ões) de pele com alteração de sensibilidade, acometimento de nervo(s) com espessamento neural e baciloscopia positiva 18 .

São considerados casos paucibacilares aqueles correspondentes às formas clínicas indeterminada e tuberculóide, e multibacilares os correspondentes às formas dimorfa e virchowiana 18. A partir de 1998, com o processo de descentralização para as unidades de saúde da família, a Área Técnica de Dermatologia Sanitária do Ministério da Saúde passa a recomendar a classificação simplificada proposta pela OMS: paucibacilar, até 5 lesões de pele, e multibacilar mais de 5 lesões. A baciloscopia, como exame complementar, se positiva, classifica o caso como multibacilar, independente do número de lesões de pele 19 .

Neste trabalho, para a classificação operacional, utilizou-se a informação contida na ficha de notificação armazenada no Sistema de Informação de Agravos de Notificação (SINAN).

\section{Fonte de dados}

Os dados foram obtidos junto ao Sistema de Vigilância da Hanseníase da Secretaria de Estado da Saúde do Espírito Santo, armazenados na base de dados do SINAN. Os dados demográficos foram os do Instituto Brasileiro de Geografia e Estatística (IBGE; http://www.ibge.gov.br) e da Rede
Interagencial de Informações para a Saúde, que se encontram disponibilizados na página de Internet do Departamento de Informática do SUS (DATASUS; http://www.datasus.gov.br).

Esta pesquisa foi aprovada pela Comissão de Ética da Faculdade de Saúde Pública da Universidade de São Paulo.

\section{Análise dos dados}

As informações relativas aos casos de hanseníase do Estado do Espírito Santo, referentes ao período de interesse, estavam armazenadas eletronicamente na base de dados do SINAN. Primeiramente foi examinada a consistência dos dados, o grau de preenchimento dos campos a serem utilizados, inclusive verificando a existência de duplicidade de notificações. Para a análise foram usados os programas Epi Info versão 6.0 (Centers for Disease Control and Prevention, Atlanta, Estados Unidos), Excel (Microsoft Corp., Estados Unidos), SPSS versão 8.0 (SPSS Inc., Chicago, Estados Unidos) e SINANW versão 5.0 (Departamento de Informática do SUS, Brasília, Brasil).

As variáveis analisadas foram os caracteres sócio-demográficos (sexo, idade), classificação operacional (multibacilares e paucibacilares) e grau de incapacidade à época do diagnóstico. Os indicadores epidemiológicos e operacionais e os parâmetros utilizados são os propostos pelo Ministério da Saúde 18 e pela International Federation of Anti-Leprosy Association (ILEP) 20.

Para a análise da tendência da hanseníase foram calculadas as taxas anuais de detecção geral, por faixa etária e por classificação operacional, para o período de 1980 a 2003. As taxas anuais foram padronizadas para idade, aplicando-se o método direto. A população utilizada como padrão foi a do Estado do Espírito Santo estimada para o ano de 2003 (http:/ /www.datasus.gov.br).

Para a análise da tendência da incidência foram utilizados modelos de regressão polinomial para séries temporais. As variáveis dependentes (Y) para cada análise foram os logaritmos das taxas anuais de detecção de hanseníase e os logaritmos das taxas anuais de detecção por faixa etária, e as variáveis independentes foram os anos calendário do período. A análise inicial incluiu a elaboração dos diagramas de dispersão gerados pelo programa Stata 7.0 (Stata Corp., College Station, Estados Unidos), entre as taxas anuais de detecção observadas (Y) e os anos do período, para definir a função que melhor expressasse a relação entre eles.

Com a finalidade de evitar autocorrelação serial entre os termos das equações de regressão, optou-se por não trabalhar com os valores do ano e sim com ano menos o ponto médio da sé- 
rie histórica. Portanto, o intervalo de tempo entre cada ano da série histórica (1980 a 2003) e o seu ponto médio (1991), foi representado pela variável centralizada $\mathrm{X}=$ ano-1991. Assim, os anos foram codificados de $-11 \mathrm{a}+12$ 21,22.

Em seguida foi realizada a modelagem, testando-se os modelos de regressão polinomial linear de primeira, segunda e de terceira ordens, representados pelas seguintes equações matemáticas:

i) $\log Y=\beta_{0}+\beta_{1} X$;

ii) $\log Y=\beta_{0}+\beta_{1} X+\beta_{2} X^{2}$;

iii) $\log Y=\beta_{0}+\beta_{1} X+\beta_{2} X^{2}+\beta_{3} X^{3}$

em que $\beta_{0}, \beta_{1}, \beta_{2}$ e $\beta_{3}$ são os coeficientes de regressão, sendo que $\beta_{0}$ representa o coeficiente médio do período e os demais os incrementos médios anuais. $\mathrm{O}$ modelo polinomial tem como objetivo encontrar a equação de regressão que melhor descreve a relação existente entre a variável independente $(\mathrm{X})$ e a variável dependente (Y).

A escolha do modelo que apresentou melhor ajuste foi baseada na análise do diagrama de dispersão, no valor do coeficiente de determinação $\mathrm{R}^{2}$ (quanto mais próximo de 1 , mais ajustado en- contra-se o modelo), na análise de resíduos e nos resultados dos testes t para cada componente do modelo. A significância estatística da tendência foi admitida quando o modelo obteve probabilidade máxima de erro de $5 \%(\mathrm{p}<0,05)$.

Em seguida, o período foi dividido em três fases, compostas de oito anos cada, para determinar as tendências de cada uma delas: estacionária, crescente ou decrescente. Foi utilizada análise de regressão, e o processamento estatístico das séries temporais foi realizado usando-se o programa Excel.

\section{Resultados}

Foram incluídos no estudo 31.030 casos segundo critérios estabelecidos pela pesquisa, sendo que destes, 22.657 eram casos novos. A trajetória das taxas de detecção de casos novos e da prevalência no período de interesse está apresentada na Figura 1. A Tabela 1 apresenta a distribuição, número e percentual dos casos novos quanto ao sexo, faixa etária e grau de incapacidade no diagnóstico.

Figura 1

Taxas de prevalência e de detecção da hanseníase. Estado do Espírito Santo, Brasil, 1980 a 2003.

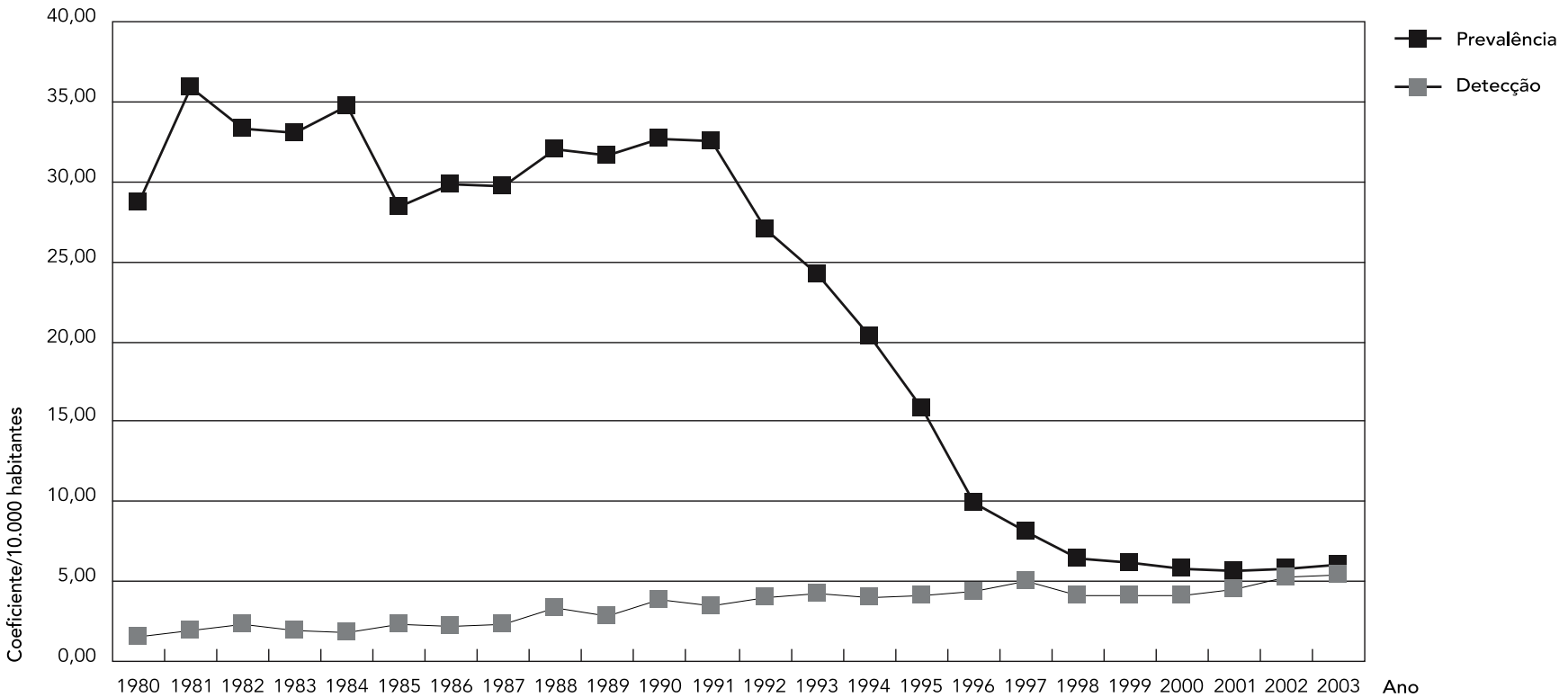

Fonte: Programa de Controle da Hanseníase, Secretaria de Estado da Saúde do Espírito Santo (prevalência), Sistema de Informação de Agravos de Notificação, Secretaria de Estado da Saúde do Espírito Santo (detecção e número de casos) e Instituto Brasileiro de Geografia e Estatística (população). 
Tabela 1

Distribuição percentual dos casos novos de hanseníase segundo faixa etária, sexo e grau de incapacidade no diagnóstico. Estado do Espírito Santo, Brasil, 1980 a 2003

\begin{tabular}{|c|c|c|c|c|c|c|c|c|c|c|c|}
\hline \multirow[t]{3}{*}{ Ano } & \multicolumn{10}{|c|}{ Faixa etária (anos) } & \multirow{3}{*}{$\begin{array}{c}\text { Ignorado } \\
n\end{array}$} \\
\hline & \multicolumn{2}{|c|}{$0-14$} & \multicolumn{2}{|c|}{$15-19$} & \multicolumn{2}{|c|}{$20-29$} & \multicolumn{2}{|c|}{$30-49$} & \multicolumn{2}{|c|}{50 e +} & \\
\hline & $\mathbf{n}$ & $\%$ & $\mathbf{n}$ & $\%$ & $\mathbf{n}$ & $\%$ & $\mathbf{n}$ & $\%$ & $\mathrm{n}$ & $\%$ & \\
\hline 1980 & 20 & 6,7 & 13 & 4,3 & 63 & 21,0 & 115 & 38,3 & 89 & 29,7 & 5 \\
\hline 1981 & 25 & 6,5 & 34 & 8,9 & 70 & 18,2 & 163 & 42,4 & 92 & 24,0 & 4 \\
\hline 1982 & 26 & 5,7 & 21 & 4,6 & 101 & 22,3 & 168 & 37,1 & 137 & 30,2 & 28 \\
\hline 1983 & 21 & 5,1 & 29 & 7,1 & 101 & 24,6 & 145 & 35,4 & 114 & 27,8 & 5 \\
\hline 1984 & 28 & 7,3 & 25 & 6,5 & 76 & 19,8 & 137 & 35,7 & 118 & 30,7 & 10 \\
\hline 1985 & 35 & 6,7 & 30 & 5,7 & 89 & 17,0 & 219 & 41,7 & 152 & 29,0 & 7 \\
\hline 1986 & 33 & 6,3 & 24 & 4,6 & 93 & 17,9 & 216 & 41,5 & 154 & 29,6 & 6 \\
\hline 1987 & 40 & 7,5 & 37 & 6,9 & 80 & 15,0 & 215 & 40,3 & 161 & 30,2 & 7 \\
\hline 1988 & 61 & 7,5 & 74 & 9,1 & 136 & 16,8 & 326 & 40,2 & 213 & 26,3 & 8 \\
\hline 1989 & 56 & 8,1 & 54 & 7,8 & 117 & 16,9 & 282 & 40,7 & 184 & 26,6 & 15 \\
\hline 1990 & 75 & 7,7 & 74 & 7,6 & 123 & 12,6 & 410 & 41,9 & 296 & 30,3 & 10 \\
\hline 1991 & 74 & 8,3 & 69 & 7,8 & 123 & 13,8 & 366 & 41,1 & 258 & 29,0 & 11 \\
\hline 1992 & 92 & 8,7 & 72 & 6,8 & 158 & 14,9 & 389 & 36,8 & 347 & 32,8 & 1 \\
\hline 1993 & 83 & 7,3 & 98 & 8,6 & 169 & 14,8 & 438 & 38,5 & 351 & 30,8 & 7 \\
\hline 1994 & 101 & 9,4 & 108 & 10,0 & 180 & 16,7 & 369 & 34,3 & 318 & 29,6 & 10 \\
\hline 1995 & 76 & 6,7 & 95 & 8,4 & 215 & 18,9 & 408 & 35,9 & 342 & 30,1 & 4 \\
\hline 1996 & 108 & 8,9 & 108 & 8,9 & 202 & 16,7 & 455 & 37,7 & 334 & 27,7 & 2 \\
\hline 1997 & 115 & 8,0 & 110 & 7,7 & 244 & 17,0 & 567 & 39,5 & 400 & 27,9 & 3 \\
\hline 1998 & 83 & 7,0 & 112 & 9,4 & 252 & 21,2 & 409 & 34,5 & 331 & 27,9 & 8 \\
\hline 1999 & 79 & 6,5 & 83 & 6,8 & 252 & 20,7 & 430 & 35,3 & 374 & 30,7 & - \\
\hline 2000 & 85 & 6,6 & 92 & 7,1 & 252 & 19,5 & 461 & 35,7 & 402 & 31,1 & - \\
\hline 2001 & 78 & 5,5 & 102 & 7,2 & 267 & 18,8 & 502 & 35,3 & 472 & 33,2 & - \\
\hline 2002 & 123 & 7,3 & 124 & 7,3 & 338 & 20,0 & 582 & 34,4 & 524 & 31,0 & 1 \\
\hline 2003 & 133 & 7,6 & 131 & 7,4 & 332 & 18,9 & 606 & 34,4 & 558 & 31,7 & 4 \\
\hline \multirow[t]{3}{*}{ Ano } & \multicolumn{4}{|c|}{ Sexo } & \multicolumn{4}{|c|}{ Grau de incapacidade } & & & \\
\hline & \multicolumn{2}{|c|}{ Feminino } & \multicolumn{2}{|c|}{ Masculino } & \multicolumn{2}{|c|}{ Avaliado * } & \multicolumn{2}{|c|}{2 ** } & & & \\
\hline & $\mathbf{n}$ & $\%$ & $\mathrm{n}$ & $\%$ & $\mathbf{n}$ & $\%$ & $\mathbf{n}$ & $\%$ & & & \\
\hline 1980 & 142 & 46,6 & 163 & 53,4 & SI & $\mathrm{SI}$ & $\mathrm{SI}$ & $\mathrm{SI}$ & & & \\
\hline 1981 & 161 & 41,5 & 227 & 58,5 & $\mathrm{SI}$ & $\mathrm{SI}$ & $\mathrm{SI}$ & $\mathrm{SI}$ & & & \\
\hline 1982 & 215 & 44,7 & 266 & 55,3 & $\mathrm{SI}$ & $\mathrm{SI}$ & $\mathrm{SI}$ & $\mathrm{SI}$ & & & \\
\hline 1983 & 168 & 40,5 & 247 & 59,5 & SI & $\mathrm{SI}$ & $\mathrm{SI}$ & SI & & & \\
\hline 1984 & 188 & 47,7 & 206 & 52,3 & $\mathrm{SI}$ & $\mathrm{SI}$ & $\mathrm{SI}$ & $\mathrm{SI}$ & & & \\
\hline 1985 & 242 & 45,5 & 290 & 54,5 & SI & $\mathrm{SI}$ & $\mathrm{SI}$ & $\mathrm{SI}$ & & & \\
\hline 1986 & 244 & 46,4 & 282 & 53,6 & SI & $\mathrm{SI}$ & $\mathrm{SI}$ & $\mathrm{SI}$ & & & \\
\hline 1987 & 250 & 46,3 & 290 & 53,7 & $\mathrm{SI}$ & $\mathrm{SI}$ & $\mathrm{SI}$ & $\mathrm{SI}$ & & & \\
\hline 1988 & 404 & 49,4 & 414 & 50,6 & SI & $\mathrm{SI}$ & $\mathrm{SI}$ & SI & & & \\
\hline 1989 & 362 & 51,1 & 346 & 48,9 & SI & $\mathrm{SI}$ & $\mathrm{SI}$ & SI & & & \\
\hline 1990 & 502 & 50,8 & 486 & 49,2 & $\mathrm{SI}$ & $\mathrm{SI}$ & $\mathrm{SI}$ & $\mathrm{SI}$ & & & \\
\hline 1991 & 416 & 46,2 & 485 & 53,8 & SI & $\mathrm{SI}$ & $\mathrm{SI}$ & SI & & & \\
\hline 1992 & 505 & 47,7 & 554 & 52,3 & SI & $\mathrm{SI}$ & $\mathrm{SI}$ & $\mathrm{SI}$ & & & \\
\hline 1993 & 541 & 47,2 & 605 & 52,8 & SI & SI & $\mathrm{SI}$ & SI & & & \\
\hline 1994 & 540 & 49,7 & 546 & 50,3 & 911 & 83,9 & 60 & 6,6 & & & \\
\hline 1995 & 550 & 48,2 & 590 & 51,8 & 982 & 86,1 & 69 & 7,0 & & & \\
\hline 1996 & 584 & 48,3 & 625 & 51,7 & 1.166 & 96,4 & 69 & 5,9 & & & \\
\hline 1997 & 658 & 45,7 & 781 & 54,3 & 1.331 & 92,5 & 95 & 7,1 & & & \\
\hline 1998 & 589 & 49,3 & 606 & 50,7 & 1.020 & 85,4 & 70 & 6,9 & & & \\
\hline
\end{tabular}

(continua) 


\begin{tabular}{|c|c|c|c|c|c|c|c|c|}
\hline \multirow[t]{3}{*}{ Ano } & \multicolumn{4}{|c|}{ Sexo } & \multicolumn{4}{|c|}{ Grau de incapacidade } \\
\hline & \multicolumn{2}{|c|}{ Feminino } & \multicolumn{2}{|c|}{ Masculino } & \multicolumn{2}{|c|}{ Avaliado * } & \multicolumn{2}{|c|}{2 ** } \\
\hline & n & $\%$ & $n$ & $\%$ & $n$ & $\%$ & $\mathrm{n}$ & $\%$ \\
\hline 1999 & 600 & 49,3 & 618 & 50,7 & 1.026 & 84,2 & 65 & 6,3 \\
\hline 2000 & 624 & 48,3 & 668 & 51,7 & 1.188 & 92,0 & 74 & 6,2 \\
\hline 2001 & 684 & 48,1 & 737 & 51,9 & 1.253 & 88,2 & 81 & 6,5 \\
\hline 2002 & 861 & 50,9 & 831 & 49,1 & 1.512 & 89,4 & 72 & 4,8 \\
\hline 2003 & 856 & 48,5 & 908 & 51,5 & 1.547 & 87,7 & 97 & 6,3 \\
\hline
\end{tabular}

SI: sem informação.

* Grau avaliado: proporção de casos novos de hanseníase que foram avaliados quanto ao grau de incapacidade física por ocasião do diagnóstico (avaliação neurológica);

** Grau 2: corresponde a casos com alguma incapacidade física.

Os modelos que melhor se ajustaram na análise de tendência da taxas de detecção de hanseníase, geral, por faixa etária e classificação dos casos em multibacilares e paucibacilares, no Estado do Espírito Santo, de 1980 a 2003, são apresentados na Tabela 2. Os resultados, tanto para a taxa de detecção geral quanto por faixa etária, com exceção da faixa de 20 a 29 anos, apontam tendência crescente, estabilizando ao final ( $\mathrm{p}<$ $0,05)$. Na faixa etária de 20 a 29 anos, a tendência mostra crescimento constante $(\mathrm{p}<0,05)$. Quanto à classificação operacional, há uma tendência de crescimento dos casos paucibacilares. Já para os casos multibacilares, o comportamento aparenta estabilizar-se ao final do período $(\mathrm{p}<0,05)$.

$\mathrm{Na}$ análise da tendência por períodos, tanto a taxa de detecção geral como por faixa etária seguiu dois padrões: estacionária ou crescente. A Figura 2 mostra as curvas de detecção por períodos de tempo: 1980 a 1987, 1988 a 1995 e 1996 a 2003. Observou-se tendência crescente significativa ( $\mathrm{p}<0,05)$ no período 1980 a 1987, para as taxas em menores de 15 anos (incremento de $7 \%$ ao ano) e 50 anos e mais, aumento de $4,8 \%$ ao ano. Nas outras faixas, a tendência mostrouse estacionária $(\mathrm{p}>0,05)$. Para o período 1988 a 1995, a tendência crescente ocorreu para a taxa de detecção geral ( $4 \%$ ao ano), e para as faixas etárias de 15 a 19 anos (6\% ao ano), 20 a 29 (6\% ao ano) e 50 anos e mais ( $6 \%$ ao ano). Nas faixas, menores de 15 anos e 30 a 49 anos, mostrou-se estacionária. No período 1996 a 2003, a tendência foi crescente apenas para a faixa etária de 20 a 29 anos (3,6\% ao ano), estando estacionária para as demais faixas e para a taxa de detecção geral.

Quanto à classificação dos casos em multibacilares e paucibacilares, houve crescimento significativo dos paucibacilares nos períodos 1980 a 1987, incremento médio anual de 6,2\%, e 1996 a 2003, incremento de $5 \%$ ( $p<0,05$ ), enquanto que, entre os multibacilares, o aumento ocorreu no período de 1988 a 1995, em média de $11 \%$ ao ano (Figura 3).

Tabela 2

Modelos que apresentaram melhor ajuste na análise de tendência das taxas de detecção de hanseníase no Estado do Espírito Santo, Brasil, 1980 a 2003.

\begin{tabular}{|c|c|c|c|c|}
\hline Taxa de detecção & Modelo & $\mathbf{R}^{2}$ & Valor de $\mathrm{p}$ & Tendência \\
\hline Geral & $\mathrm{Y}=\mathrm{e} 3,62861+0,0447886 \mathrm{X}+(-0,0018703) \mathrm{X} 2$ & 0,88 & 0,004 & Crescente estabilizando ao final \\
\hline $0-14$ anos & $Y=e 2,031885+0,0731446 X+(-0,0032846) X 2$ & 0,89 & 0,002 & Crescente estabilizando ao final \\
\hline 15-19 anos & $Y=e 3,248161+0,0744476 X+(-0,0046559) X 2$ & 0,84 & 0,001 & Crescente estabilizando ao final \\
\hline $20-29$ anos & $Y=e 3,416142+0,0525496 X$ & 0,89 & $<0,0001$ & Crescente \\
\hline $30-49$ anos & $\mathrm{Y}=\mathrm{e} 4,02571+0,0326687 \mathrm{X}+(-0,0022544) \mathrm{X} 2$ & 0,74 & 0,004 & Crescente estabilizando ao final \\
\hline 50 anos e + & $Y=e 4,361854+0,0463361 X+(-0,0020057) X 2$ & 0,87 & 0,004 & Crescente estabilizando ao final \\
\hline Paucibacilar & $Y=e 2,827431+0,0536383 X$ & 0,84 & $<0,0001$ & Crescente \\
\hline Multibacilar & $\mathrm{Y}=\mathrm{e} 2,81015+0,0487861 \mathrm{X}+(-0,0030865) \mathrm{X} 2$ & 0,75 & 0,006 & Crescente estabilizando ao final \\
\hline
\end{tabular}


Figura 2

Tendência das taxas de deteç̧ão de hanseníase (por 100 mil habitantes) por faixa etária. Estado do Espírito Santo, Brasil, 1980 a 2003.

2a) Geral

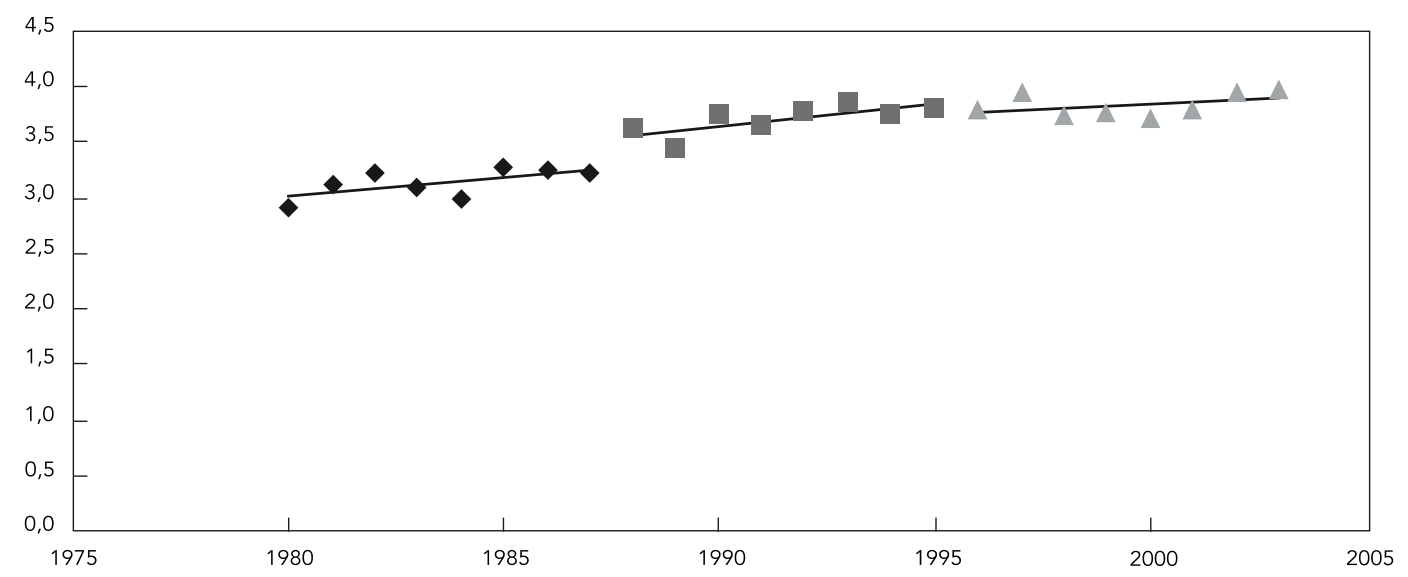

2b) 0-14 anos

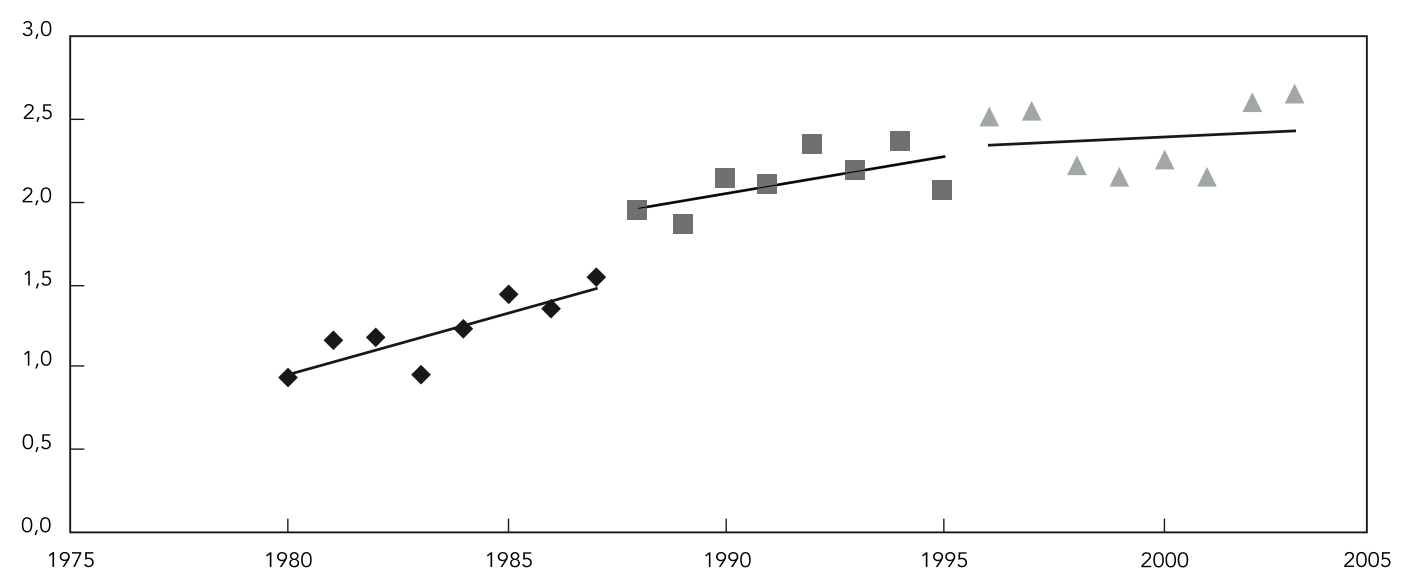

2c) 15-19 anos

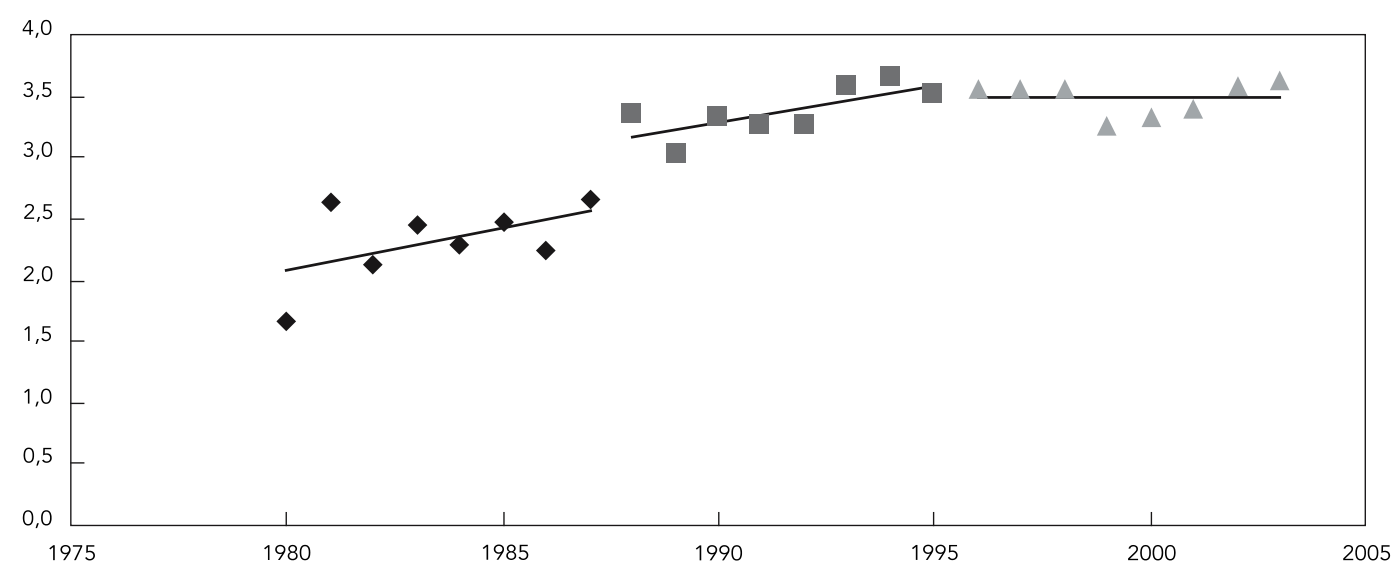

(continua) 
Figura 2 (continuação)

2d) 20-29 anos

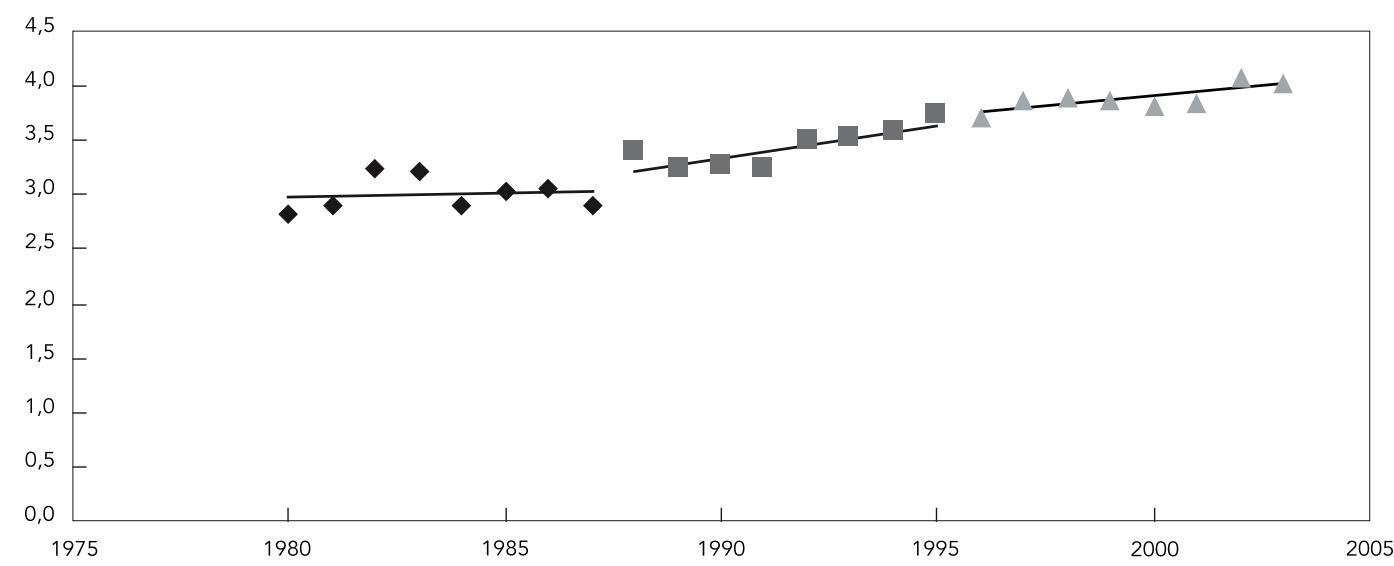

2e) 30-49 anos

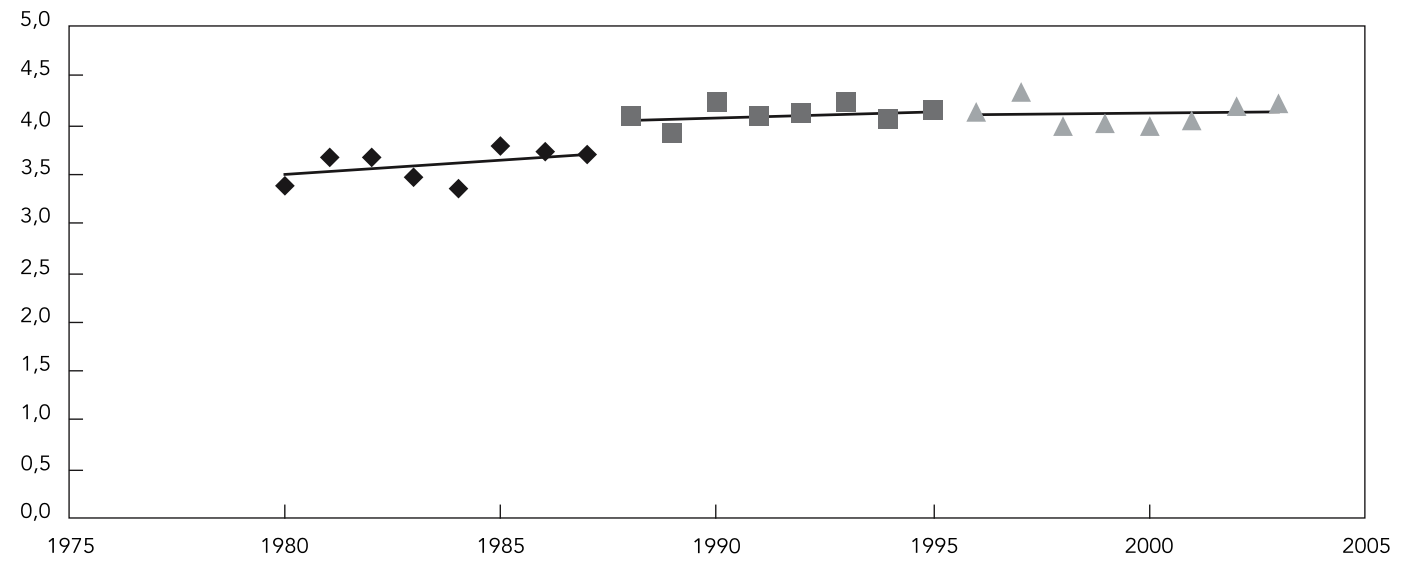

2f) 50 anos e +

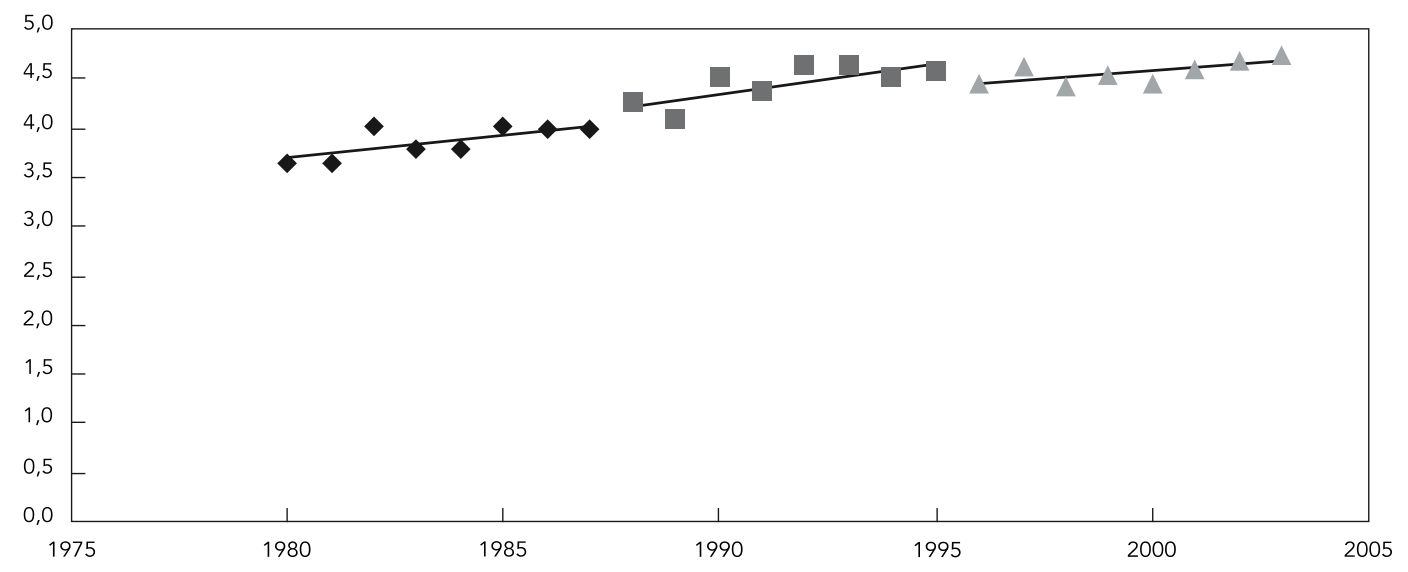


Tendência das taxas de detecção da hanseníase (por 100 mil habitantes) segundo classificação operacional. Estado do Espírito Santo, Brasil, 1980 a 2003.

3a) Paubacilar

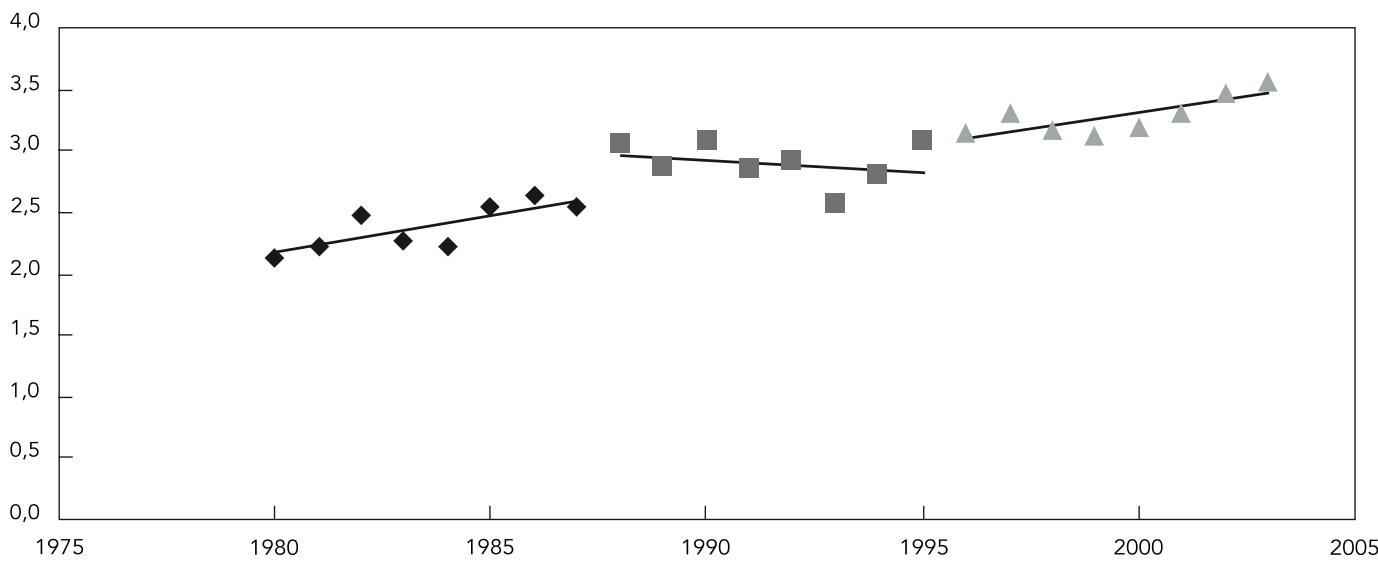

3b) Multibacilar

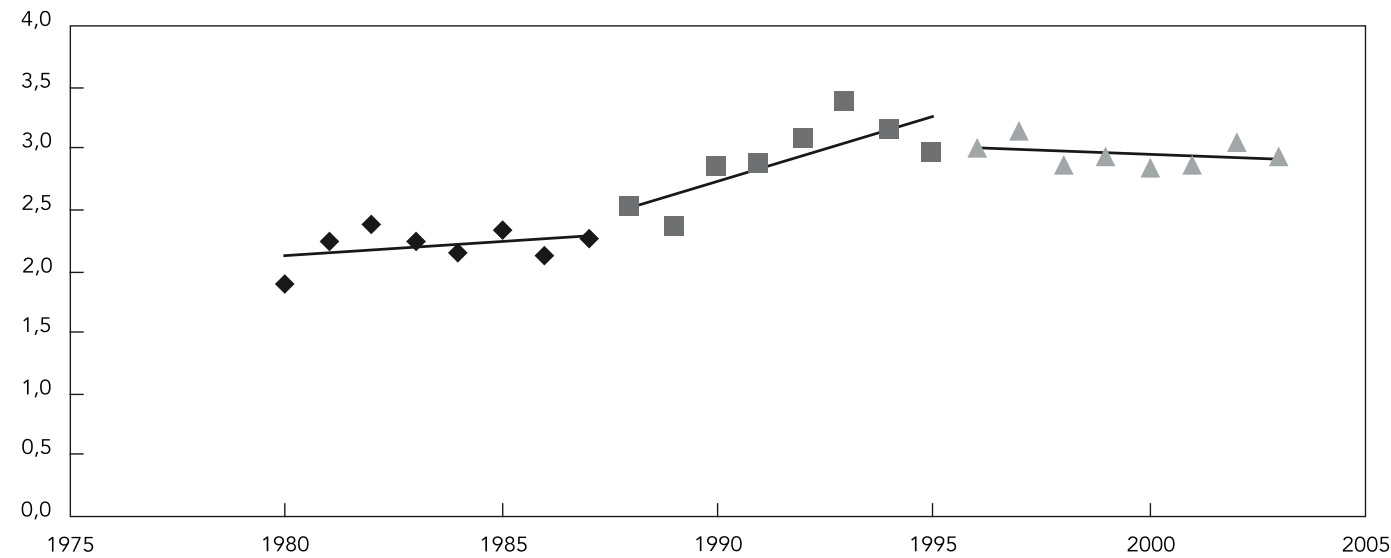

Na distribuição percentual por sexo, há um predomínio discreto de acometimento do sexo masculino para quase todo o período, em média 1,1 homem para uma mulher.

Quanto à faixa etária, a proporção de casos novos em menores de 15 anos iniciou o período com $6,7 \%$, atingiu seu pico máximo em 1994 , de 9,4\%, caindo a partir de então, chegando em 2003 a 7,6\%. Para a faixa de 15 a 19 anos, o desempenho é semelhante à de menores de 15 anos. Ao mesmo tempo, os percentuais para a faixa de 50 anos e mais vêm sofrendo um aumento discreto, tendo alcançado seu maior índice, $33,2 \%$, em 2001 e seu menor índice, 24,0\%, em 1981. Perce- be-se também queda discreta nos percentuais de 20 a 29 anos e de 30 a 49 (Tabela 1).

A informação quanto ao grau de incapacidade só tornou-se consistente a partir de 1994, com $83,9 \%$ dos casos avaliados, chegando a $87,7 \%$ em 2003. Quanto ao grau de incapacidade no diagnóstico, o grau 2 de incapacidade mostra discretas alterações, estando na maioria do período em torno de $6 \%$ (Tabela 1 ).

O abandono de tratamento, para o período 1994 a 2003, caiu de $45,2 \%$ em 1994 para $5 \%$ em 2003, atingindo seu menor índice em 2001, com $3,4 \% 15$. 


\section{Discussão}

Para melhor compreensão dos resultados apresentados, cabe salientar algumas limitações do estudo, dentre elas, mudanças de definições aplicadas na classificação operacional, ocorridas no período de interesse 6,19,20,23 , e variações da sensibilidade do sistema de vigilância e das características das fontes de informação 4,23, a partir do início dos anos 90 com a introdução da poliquimioterapia.

A prevalência sofre declínio acentuado entre os anos de 1990 e 1996, coincidindo com o incremento da poliquimioterapia no estado. Este regime de tratamento trouxe mudanças estruturais ao acompanhamento da endemia, principalmente nas normas para se proceder as altas por cura e para permanência do doente em abandono no registro ativo 24,25 . O encurtamento do tempo de tratamento, com maior adesão do paciente, refletiu em incremento das altas por cura, e em decorrência da diminuição do tempo de abandono para se proceder a altas estatísticas (saídas do registro por abandono), foram reavaliados os registros dos pacientes, com revisão cuidadosa do arquivo, repercutindo no aumento deste tipo de saída 24.

Quanto aos casos novos, nossos resultados mostram um crescimento constante das taxas de detecção, durante o período do estudo, tendência semelhante à verificada em outras regiões do Brasil 6,8,12,26, ainda que menos acentuada a partir de 1996. Essas taxas, segundo parâmetros do Programa Nacional de Controle da Hanseníase, situam o Estado do Espírito Santo entre as Unidades da Federação aonde a hanseníase apresenta-se em níveis hiperendêmicos, ou seja, com taxas iguais ou maiores que 4,0/10 mil habitantes 10 .

O crescimento mais acentuado no período de 1988 a 1995, coincide com a implantação da poliquimioterapia 27,28 que resultou na introdução de uma nova estratégia do programa pelos serviços, induzindo um aumento da sensibilidade da vigilância 29 , fato que deve explicar em boa parte o crescimento assinalado.

Entre as medidas mais marcantes que precederam e/ou coincidiram com a introdução da poliquimioterapia no Estado do Espírito Santo temos o início da municipalização dos serviços, que atualmente abrange todo o estado; maior padronização das ações de controle da hanseníase; parceria com a sociedade civil organizada e organizações não-governamentais ${ }^{3}$ como a Netherland Leprosy Relief (NLR), propiciando maior aporte de recursos financeiros; intensificação das atividades de educação sanitária visando à descoberta de casos, com a realização do "Dia da Mancha", de âmbito estadual, e várias campanhas municipais 3 ; supervisões sistemáticas em todos os municípios ao menos duas vezes ao ano; monitoramento do sistema de informações, incluindo medidas visando a conferir maior validade aos dados disponíveis 11 .

Com a implantação da Estratégia de Saúde da Família em 1998, o Estado do Espírito Santo investiu em treinamento das equipes do programa e dos agentes comunitários de saúde, com vistas à descentralização, tendo hoje várias equipes desenvolvendo ações do programa, com suporte de um sistema de referência municipal e de um centro de referência estadual para as intercorrências 15 .

Foram realizadas parcerias com universidades por meio de projetos ensino/serviço, com professores e alunos atuando em escolas de primeiro e segundo graus, comunidades, e no desenvolvimento de pesquisas, visando, além da busca ativa de casos, à capacitação dos estudantes de graduação.

Esse conjunto de medidas, fortalecendo o programa em todo o estado, é consistente com nossa hipótese de que o aumento de casos novos pode estar em grande parte relacionado ao aperfeiçoamento do programa 3,20 .

A taxa de detecção crescente entre menores de 15 anos e na faixa etária de 15 a 19, indica a manutenção de níveis elevados de transmissão recente da doença $11,17,23$. Segundo parâmetros do ILEP, $10 \%$ parece ser o limite entre o que é alta ou baixa proporção de casos novos descobertos em menores de 15 anos 20 . Como dado favorável, verificamos que de 1994 a 2003 houve uma diminuição dessa taxa, concomitante a ampliação das atividades de busca ativa nessa faixa etária, apontando um maior controle da doença 30.

Estudo sobre a expansão da endemia no nordeste brasileiro encontrou aumento na detecção de casos em grupos etários mais velhos, provavelmente resultado de casos acumulados, descobertos com a intensificação das atividades de detecção 20,31. No Espírito Santo, para a faixa etária de 50 anos e mais, esse aumento foi mais intenso no período 1988 a 1995, coincidindo com o incremento dessas atividades.

Segundo relatos da literatura, a idade para o aparecimento das manifestações clínicas da enfermidade é em geral a do adulto jovem ${ }^{6}$. Observa-se que a incidência da doença no estado apresentou tendência crescente no último período apenas para a faixa etária de 20 a 29 anos. Esse dado pode sugerir precocidade do diagnóstico, o que se coaduna com as atividades desenvolvidas.

Os resultados encontrados na distribuição por sexo não diferem dos registrados em outros 
estudos 12,20,23, com predomínio discreto do sexo masculino.

Quanto à classificação operacional, paucibacilares e multibacilares, a tendência destes últimos entre os casos novos aumenta no início das atividades de um programa 20 . No decorrer do processo, quando o programa passa para uma fase intermediária, essa proporção tende a se reduzir com o incremento da detecção de casos paucibacilares, fato que também pode ocorrer quando temos campanhas com busca ativa 20 . Nossos resultados mostram-se consistentes com essa análise. O período de crescimento dos multibacilares, 1988 a 1995, coincide com a implementação das ações. Já os paucibacilares, apresentaram tendência crescente de 1980 a 1987 e de 1996 a 2003. Como dito anteriormente, na última década intensificaram-se as atividades de busca ativa de casos.

O grau de incapacidade à época do diagnóstico é um dos parâmetros mais utilizados para avaliar a sua precocidade 9 . Somente a partir de 1994, passamos a ter essa informação para mais de $75 \%$ dos casos novos detectados, índice que segundo normas do Ministério da Saúde 18 permite avaliar a proporção de casos com incapacidade. Os resultados verificados a partir de 1994, apontam patamares próximos aos considerados como baixos, ou seja, em torno de $5 \% 20$, sugerin- do que o diagnóstico está sendo efetuado precocemente.

O abandono de tratamento é um indicador utilizado na avaliação da capacidade dos serviços em assistir aos casos de hanseníase ${ }^{18}$. No Espírito Santo, na última década, este percentual caiu de forma expressiva, atingindo 5\%, em 2003. Desde o ano de 1997, esse percentual não ultrapassa o limite considerado bom 18 , ou seja, em torno de $10 \%$. Tais resultados, possivelmente estejam relacionados ao maior acompanhamento dos municípios pelo estado com vistas a atingir as metas estabelecidas nos Pacto da Atenção Básica e da Programação Pactuada e Integrada, de diminuir a proporção de abandono de tratamento e o grau de incapacidade à época do diagnóstico, ampliar o percentual de cura e o de casos novos descobertos em relação ao esperado segundo estudo de tendência 15 .

Em síntese, os resultados deste trabalho mostram que além das medidas já tomadas pelo Programa Estadual de Controle da Hanseníase e citadas neste texto, é importante o desenvolvimento de estudos que nos permitam melhor conhecer os mecanismos de transmissão mais relevantes nessa fase de controle da doença, assim como para identificar grupos de maior risco para constituírem resíduos de fontes de infecção, especialmente no ambiente domiciliar. 


\section{Resumo}

Descrição do comportamento e análise da tendência da hanseníase entre pacientes residentes no Estado do Espírito Santo, Brasil, de 1980 a 2003. Utilizando modelos estatísticos para séries temporais, identificou-se tendência crescente para todo o período da taxa de detecção global $(p<0,05)$ com aparente estabilização no final do período, verificamos também tendência crescente para os períodos: (i) 1980-1987 nos grupos etários de $<15$ anos e 50 anos e mais e para formas paucibacilares; (ii) 1988-1995 para as faixas de 15-19 anos, 20-29 e 50 anos e mais e para formas multibacilares; (iii) 1996-2003 no grupo de 20-29 anos e formas paucibacilares. Os indicadores de avaliação da endemia apontaram patamares estáveis do grau de incapacidade 2 (em média 6\%); a proporção de casos entre $<15$ anos situou-se abaixo de $10 \%$ e a de abandono de tratamento em torno de 6\%. A prevalência apresentou forte declínio. A tendência crescente pode ser explicada, em parte pela maior sensibilidade da vigilância, mas a elevada proporção entre $<15$ anos aponta a necessidade de estudos visando ao melhor conhecimento dos resíduos de fontes de infecção especialmente no domicílio.

Hanseníase; Doenças Endêmicas; Estudos de Séries Temporais

\section{Referências}

1. Helene LMF, Salum MJL. A reprodução social da hanseníase: um estudo do perfil de doentes com hanseníase no Município de São Paulo. Cad Saúde Pública 2002; 18:101-13.

2. Lockwood DNJ. Commentary: leprosy and poverty. Int J Epidemiol 2004; 33:269-70.

3. Lockwood DNJ, Suneetha S. Leprosy: too complex disease for a simple elimination paradigm. Bull World Health Organ 2005; 83:230-5.

4. Opromolla PA, Dalben I, Cardim M. Análise da distribuição espacial da hanseníase no Estado de São Paulo, 1991-2002. Rev Bras Epidemiol 2005; 8:356-64.

5. Área Técnica de Dermatologia Sanitária, Secretaria de Vigilância em Saúde, Ministério da Saúde. Prevalência e detecção da hanseníase, segundo Unidade Federada - Brasil, 2003. Brasília: Ministério da Saúde; 2004.

6. Martelli CMT, Stefani MMA, Penna GO, Andrade ALSS. Endemias e epidemias brasileiras, desafios e perspectives de investigação científica: hanseníase. Rev Bras Epidemiol 2002; 5:273-85.

\section{Colaboradores}

M. V. Moreira coordenou a pesquisa e participou de todas as suas etapas, elaboração do projeto, análise de dados e redação do artigo. E. A. Waldman participou da análise de dados e redação do artigo. C. L. Martins orientou o desenvolvimento da pesquisa, participou da efetuação da análise assim como da redação do artigo.

\section{Agradecimentos}

Agradecimento especial ao Dr. Diltor Vladimir Araújo Opromolla (in memorian), que muito contribuiu com suas críticas e sugestões para a conclusão de nosso trabalho. À equipe de profissionais que atuam no Programa de Controle e Eliminação da Hanseníase da Secretaria de Estado da Saúde do Espírito Santo, em especial à Enfermeira Marizete Altoé Puppim e Dra. Elizabete Santos Madeira.
7. Matos HJ, Duppre N, Alvim MFS, Vieira LMM, Sarno EM, Struchner CJ. Epidemiologia da hanseníase em coorte de contatos intradomiciliares no Rio de Janeiro (1987-1991). Cad Saúde Pública 1999; 15:535-42

8. Lapa T, Ximenes R, Silva NN, Souza W, Albuquerque MFM, Campozana G. Vigilância da hanseníase em Olinda, Brasil, utilizando técnicas de análise espacial. Cad Saúde Pública 2001; 17:1153-62.

9. Magalhães MCC, Rojas LI. Evolución de la endemia de la lepra en Brasil. Rev Bras Epidemiol 2005; 8:342-55.

10. Rede Interagencial de Informações para a Saúde, Ministério da Saúde. Indicadores e dados básicos para a saúde. http://www.datasus.gov.br (acessado em 31/Out/2004).

11. Lana FCF, Lima RF, Araújo MG, Fonseca PTS. Situação epidemiológica da hanseníase no Município de Belo Horizonte/MG - período 92/97. Hansen Int 2000; 25:121-32. 
12. Figueiredo IA, Silva AAM. Aumento da detecção de casos de hanseníase em São Luís, Maranhão, Brasil, de 1993 a 1998. A endemia está em expansão? Cad Saúde Pública 2003; 19:439-45.

13. Aquino DMC, Santos JS, Costa JML. Avaliação do programa de controle da hanseníase em um município hiperendêmico do Estado do Maranhão, Brasil, 1991-1995. Cad Saúde Pública 2003; 19:119-25.

14. Cunha SS, Rodrigues LC, Duppre NC. Current strategy for leprosy control in Brazil: time to pursue alternativa preventive strategies? Pan Am J Public Health 2004; 16:362-5.

15. Superintendência de Planejamento Epidemiologia e Informação, Secretaria de Estado da Saúde do Espírito Santo. Programa estadual de controle da hanseníase no Espírito Santo. Hanseníase - dados epidemiológicos - ano 2003. Vitória: Secretaria de Estado da Saúde do Espírito Santo; 2003.

16. Araújo MG. Hanseníase no Brasil. Rev Soc Bras Med Trop 2003; 36:373-82.

17. Souza WV, Barcellos CC, Brito AM, Carvalho MS, Cruz OG, Albuquerque MFM, et al. Aplicação de modelo bayesiano empírico na análise espacial da ocorrência de hanseníase. Rev Saúde Pública 2001; 35:474-80.

18. Área Técnica de Dermatologia Sanitária, Departamento de Atenção Básica, Secretaria de Políticas de Saúde, Ministério da Saúde. Hanseníase: atividades de controle e manual de procedimentos. Brasília: Ministério da Saúde; 2001.

19. Gallo MEN, Ramos Jr. LAN, Albuquerque ECA, Nery JAC, Sales AM. Alocação do paciente hanseniano na poliquimioterapia: correlação da classificação baseada no número de lesões cutâneas com os exames baciloscópicos. An Bras Dermatol 2003; 78:415-24.

20. International Federation of Anti-Leprosy Association. A interpretação dos indicadores epidemiológicos da lepra [CD-ROM]. London: International Federation of Anti-Leprosy Association; 2002.

21. Latorre MRDO. A mortalidade por câncer de estômago no Brasil: análise do período de 1977 a 1989. Cad Saúde Pública 1997; 13 Suppl 1:S567-78.
22. Holcman MM, Latorre MRDO, Santos JLF. Evolução da mortalidade infantil na região metropolitana de São Paulo, 1980-2000. Rev Saúde Pública 2004; 38:180-6.

23. Lombardi C. Tendência secular da detecção da hanseníase no Estado de São Paulo (1934-1983). Rev Patol Trop 1993; 22:407-87.

24. Andrade VLG. Evolução da hanseníase no Brasil e perspectivas para sua eliminação como problema de saúde pública [Tese de Doutorado]. Rio de Janeiro: Escola Nacional de Saúde Pública, Fundação Oswaldo Cruz; 1996.

25. Opromolla DV, organizador. Noções de hansenologia. Bauru: Centro de Estudos Dr. Reynaldo Quaqliato; 2000.

26. Hinrichsen SL, Pinheiro MRS, Jucá MB, Rolim H, Danda GJN, Danda DMR. Aspectos epidemiológicos da hanseníase na Cidade de Recife, PE em 2002. An Bras Dermatol 2004; 79:413-21.

27. Munhoz-Jr S, Fontes CJFF, Meirelles SMP. Avaliação do programa de controle da hanseníase em municípios mato-grossenses, Brasil. Rev Saúde Pública 1997; 31:282-7.

28. Dias MCFS, Dias GH, Nobre MLN. Distribuição espacial da hanseníase no Município de Mossoró/ RN, utilizando o Sistema de Informação Geográfica - SIG. An Bras Dermatol 2005; 80 Suppl 3:S289-94.

29. Soares LS, Moreira RO, Vilela VV, Alves MJM, Pimentel AFM, Ferreira AP, et al. The impact of multidrug therapy on the epidemiological pattern of leprosy in Juiz de Fora, Brazil. Cad Saúde Pública 2000; 16:343-50.

30. Pereira Jr. AC, Torrecilla MAA. Profilaxia. In: Talhari S, Neves RG, organizadores. Dermatologia tropical: hanseníase. 3a Ed. Manaus: Editora Lorena; 1997. p. 151-2.

31. Albuquerque MFPM, Morais HMM, Ximenes $\mathrm{R}$. A expansão da hanseníase no nordeste brasileiro. Rev Saúde Pública 1989; 23:107-16.

Recebido em 23/Jan/2007

Versão final reapresentada em 06/Jul/2007

Aprovado em 03/Dez/2007 